\title{
Discharge plasma formation in square capillary with gas supply channels
}

\author{
G. A. Bagdasarov $\odot,{ }^{1}$ K. O. Kruchinin $\odot,,^{2, *}$ A. Yu. Molodozhentsev $\odot,{ }^{2}$ P. V. Sasorov $\odot,{ }^{1,2}$ \\ S. V. Bulanov $\circledast^{2,3}$ and V. A. Gasilov $\circledast^{1}$ \\ ${ }^{1}$ Keldysh Institute of Applied Mathematics RAS, 125047 Moscow, Russia \\ ${ }^{2}$ Institute of Physics ASCR v.v.i. (FZU), ELI-Beamlines, 18221 Prague, Czech Republic \\ ${ }^{3}$ National Institutes for Quantum Science and Technology (QST), Kansai Photon Science Institute, Kizugawa, Kyoto 619-0215, Japan
}

(Received 8 October 2021; accepted 13 January 2022; published 31 January 2022)

\begin{abstract}
A comprehensive model of processes in a discharge capillary is required in order to obtain nominal parameters of a preformed plasma channel suitable for the laser wake-field acceleration. We present three-dimensional magnetohydrodynamics simulations of a hydrogen gas filling process and discharge plasma formation in a short square shaped capillary with gas supply channels. Time evolution of the gas pressure and the plasma density in the capillary channel for a chosen discharge current profile is analyzed. Performed simulations provide distributions of the electric current, the magnetic field, and the electron density along the whole channel, taking into account gas supply areas as well as areas outside of the capillary. Obtained results show that the presence of gas supplies leads to the inhomogeneous plasma density distribution along the capillary channel which has to be taken into account for generating an optimal laser-driven electron beam.
\end{abstract}

DOI: 10.1103/PhysRevResearch.4.013063

\section{INTRODUCTION}

A plasma-based acceleration scheme for particle acceleration by space charge wave was proposed by Fainberg in 1956 [1]. Later on, plasma-based accelerators (PBAs) were considered as an alternative for the conventional ones. PBAs use either intense laser pulses in the case of laser wake-field acceleration (LWFA) [2] or charged particle beams passing through a plasma in the case of plasma wake-field acceleration (PWFA) [3]. These acceleration approaches allow us to overcome one of the most significant limitations in conventional accelerators-limited electric field gradient in radio frequency accelerating structures. Extreme LWFA accelerating gradients, demonstrated experimentally by different teams [4-7], offer a path towards a compact PBA needed in a broad variety of applications, including free electron lasers (FELs) [8-14], Thomson sources [15-17], and electron-positron colliders with TeV energy $[18,19]$.

Nowadays an electrical discharge in a gas filled capillary [20-27] is a common solution for plasma generation. Such devices are designed to provide specific plasma density profiles in order to generate tailored wake fields, guide the laser pulse, and focus particle beams [28-30]. The most suitable approach to create a plasma channel is an electrical discharge in a gas filled capillary [31,32]. In this case, the plasma in the channel is fully ionized, minimizing spatial or temporal distortion of

\footnotetext{
*konstantin.kruchinin@eli-beams.eu

Published by the American Physical Society under the terms of the Creative Commons Attribution 4.0 International license. Further distribution of this work must maintain attribution to the author(s) and the published article's title, journal citation, and DOI.
}

the guided laser pulse. In addition, such a channel is long lived, permitting synchronization with the laser system. A prefilled low $Z$ gas, usually hydrogen, is transformed in the capillary into an underdense plasma via avalanche breakdown and plasma heating. Subsequent thermal conduction to the capillary walls results in the temperature of the plasma being greater on axis. Since the pressure across the capillary is uniform, an axial minimum in the plasma density will be formed. For an ideal channel, the plasma density can be approximated by the parabolic function: $n(r)=n_{0}+\Delta n\left(r / R_{c}\right)^{2}$, where $n_{0}$ is the on-axis density, $r$ is the distance from the axis, $\Delta n$ is the channel depth, and $R_{c}$ is the channel radius [33]. In the absence of further ionization of the plasma by the guided laser pulse a Gaussian laser beam propagates through the channel with a constant spot size, if the matching condition is satisfied.

For accelerating electrons up to $8 \mathrm{GeV}$ a high power laser as well as a long capillary with the length of $20 \mathrm{~cm}$ with a low plasma discharge density are needed [27]. These requirements enforce the use of wider capillaries which leads to the insufficient guiding abilities of such plasma channels. To solve this problem a plasma channel heating technique was proposed [27,34-36] where the plasma channel is preformed by an additional laser pulse injected into the capillary right before the main laser pulse used for acceleration. Realization of this technique allowed for achieving the record electron energies of $\sim 8 \mathrm{GeV}$ through the laser wake-field acceleration $[27,35,36]$. For the electron acceleration up to a several hundreds of $\mathrm{MeV}$, required for an extreme ultraviolet laser-driven FEL [13], much shorter discharge capillaries with a higher plasma density can be used. Thus, no additional heating of the plasma is needed. However, in the short capillary the presence of gas supply channels is playing a significant role leading to the distortion of the uniform, symmetrical plasma channel. 
Capillary discharge aimed for utilization in PBAs were simulated both in the frame of one-dimensional (1D) and 2D geometries $[33,34,37-40]$. The process of the capillary gas filling was simulated using full 3D geometry of capillary in [38]. However, certain important details of the 3D design, in particular the square shape of the capillary and gas inlet channels, were not taken into account yet in the magnetohydrodynamics (MHD) simulations.

In this paper, we present a 3D MHD simulation of the gas filling process and the discharge formation in a capillary with square cross section using the MARPLE [41] code. A whole 3D geometry of the capillary was taken into account, including gas supply areas. Capillary geometry and gas parameters were chosen to correspond to the ones designed for the laser-driven extreme ultraviolet EUV-FEL which is currently under development at ELI-Beamlines [42].

\section{CAPILLARY PARAMETERS, BASED ON THE LWFA SCALING FOR THE EUV-FEL}

Mechanisms of the laser wake-field acceleration in the preformed plasma channel, utilizing the electrical discharge in the gas-filled capillary, are quite complex. Proper multiparametric optimization is needed in order to obtain a high quality electron beam suitable for the laser-driven FEL. As it was demonstrated experimentally, the electron self-injection and the laser-driven electron acceleration depend sensitively on a number of parameters such as a laser intensity at the focus, a plasma density in the laser-plasma interaction area, and a delay between the onset of the discharge current and the arrival of the laser pulse [43]. In the case of the laser-driven FEL the plasma density in a preformed plasma channel and the laser parameters are determined mainly by the electron beam quality, required to generate a self-amplified spontaneous emission in a commercially available undulator. In order to develop the EUV-FEL with the photon radiation wave length of around $20-30 \mathrm{~nm}$, utilizing the hybrid permanent magnet undulator (for instance, the "Swiss-FEL" type undulator [44]), the energy of the electron beam should be around 350$400 \mathrm{MeV}$ [45]. Recently, a proof-of-principal demonstration of free electron lasing at 27-nm photon wavelength, based on the 490-MeV compact laser wake-field accelerator, has been published [46]. The electron self-injection and acceleration were performed utilizing the pure helium gas flow through the nozzle with a shock front.

The LWFA scaling [47] was used in order to estimate a set of main laser and plasma parameters aiming to reach the required energy of the electron beam. The required energy range of the laser-driven electron beam can be obtained utilizing a 100-TW class laser with a laser pulse of $30 \mathrm{fs}$ and a laser wavelength of $800 \mathrm{~nm}$. According to the scaling analysis the plasma density, which depends on the laser spot size and the laser pulse power in the focus, should be around $(1.2-1.5) \times 10^{18} \mathrm{~cm}^{-3}$. The full width at half maximum spot size of the laser beam in the focus should be equal to around $30-40 \mu \mathrm{m}$. In this case the acceleration length, estimated by the dephasing length, will be limited by $5-7 \mathrm{~mm}$.

Based on the performed scaling analysis the following parameters of the sapphire capillary have been chosen in order to reach the required electron beam energy: The capillary

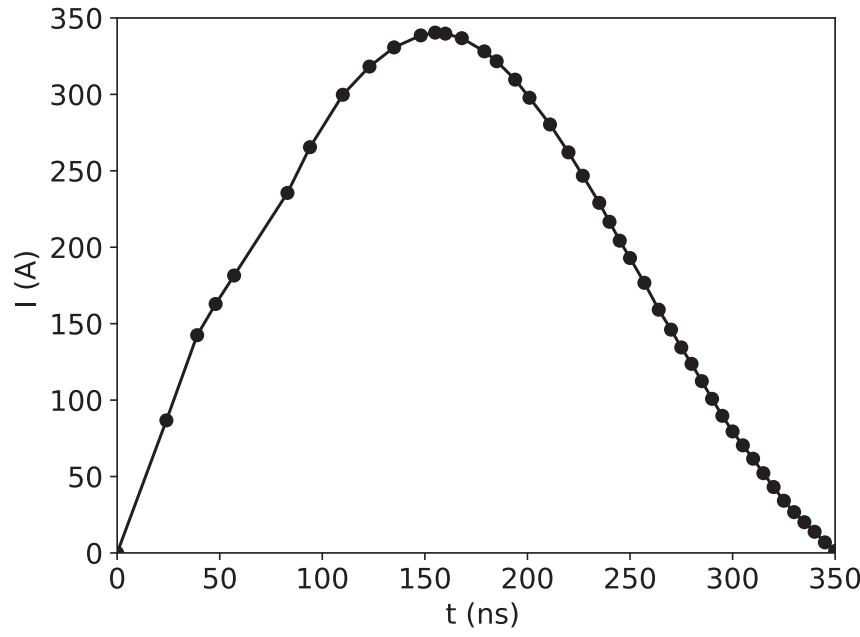

FIG. 1. Measured profile of the discharge electric current.

length is $10 \mathrm{~mm}$ and the transverse size of the square shape channel is $500 \mu \mathrm{m}$. The square shape of the capillary channel is chosen from the technological point of view. Plasma channel parameters produced in such square shaped capillary and in a circular one are the same in the vicinity of their axes and have noticeable differences only near the walls [37]. The hydrogen gas flows into the capillary via two square shaped supply channels with a transverse size of $500 \mu \mathrm{m}$ and length of $5 \mathrm{~mm}$ located $2 \mathrm{~mm}$ from each edge of the sapphire plate. The thickness of the copper electrode, mounted from both sides of the plates, is $2 \mathrm{~mm}$. The gas pressure in the gas supply channels is around $40 \mathrm{mbar}$. An electrical discharge through the capillary is driven by a high voltage pulse of up to $20 \mathrm{kV}$ producing the peak current of up to $340 \mathrm{~A}$. The measured electric current profile is shown in Fig. 1.

\section{3D MHD MODEL}

The modeling of the discharge plasma formation in the capillary channel is divided into two sequential stages. At first, we simulated the process of the capillary filling with neutral hydrogen until the steady state is reached. Second, the discharge stage is modeled using the obtained gas distribution as an initial one. The hydrodynamic model including viscosity and conductive heat transfer effects taken into account is used to simulate the first stage. A one-fluid, two-temperature (ion and electron) MHD model [48], accounting for the dissipation effects (electron and ion thermal conductivity, the electronion energy exchange, the magnetic field diffusion due to the finite electric conductivity, the Joule heating, and the radiation losses) is used to simulate the second stage. The effect of the displacement current is also preserved allowing the modeling of the magnetic field propagation process in the insulator, which is required for the accurate modeling of the magnetic field on the capillary boundary and in the orifice of the supply channel and the capillary crossing. Plasma is assumed to be weakly ionized (mean plasma charge $Z=5 \times 10^{-5}$ ) at the beginning of the second stage in order to initiate the discharge without modeling of the electrical breakdown in molecular hydrogen. The equation of state and dissipation coefficients 


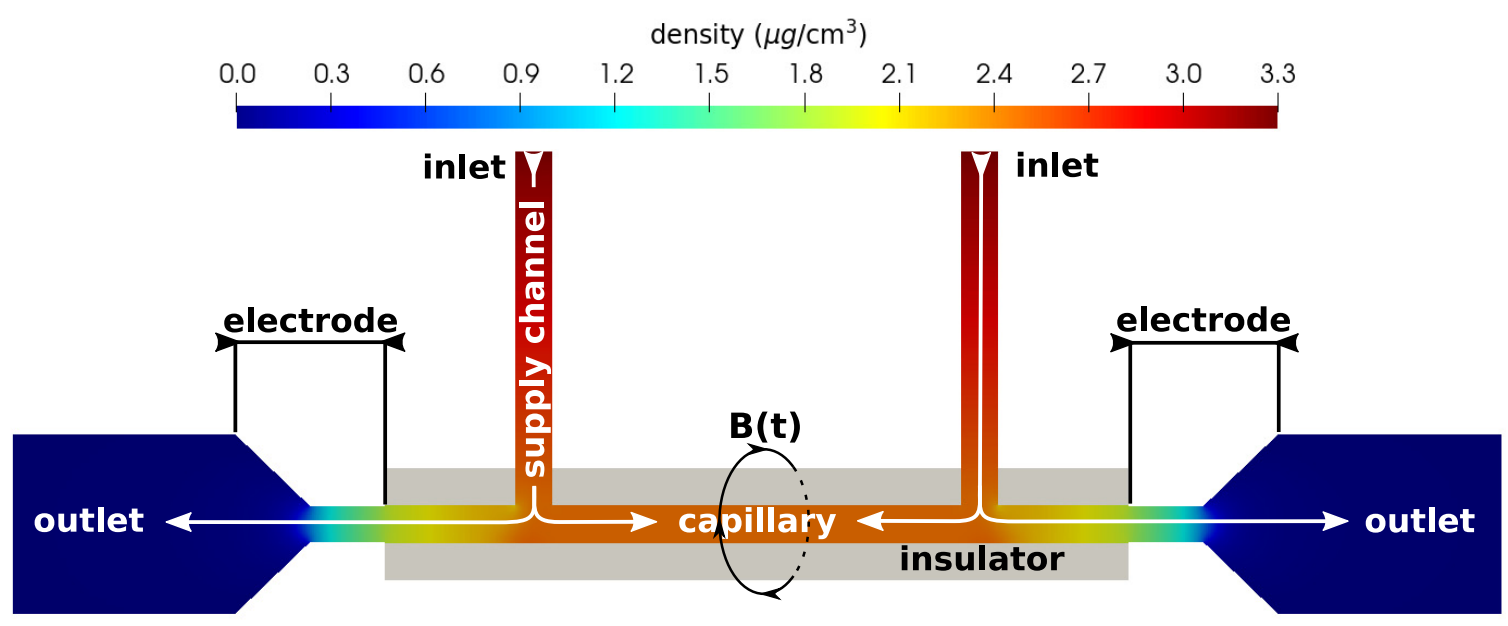

FIG. 2. Computational domain diagram for the capillary discharge simulation setup.

incorporate the degree of the gas ionization. The MHD model can be presented by the following set of equations:

$$
\begin{gathered}
\partial_{t} \rho+\nabla(\rho \mathbf{v})=0, \\
\rho\left(\partial_{t}+\mathbf{v} \nabla\right) \mathbf{v}=-\nabla p+\mathbf{j} \times \mathbf{B} / c+\mu \Delta \mathbf{v}, \\
\rho\left(\partial_{t}+\mathbf{v} \nabla\right) \varepsilon_{i}+p_{i} \nabla \mathbf{v}=\nabla\left(\kappa_{i} \nabla T_{i}\right)+Q_{e i}, \\
\rho\left(\partial_{t}+\mathbf{v} \nabla\right) \varepsilon_{e}+p_{e} \nabla \mathbf{v} \\
=\nabla\left(\kappa_{e} \nabla T_{e}\right)-Q_{e i}+Q_{\mathrm{rad}}+\mathbf{j}^{2} / \sigma+Q_{l}, \\
\partial_{t} \mathbf{B}+\nabla \times\left(v_{m} \nabla \times \mathbf{B}\right)=\nabla \times(\mathbf{v} \times \mathbf{B}), \\
\mathbf{j}=(c / 4 \pi)\left(\nabla \times \mathbf{B}+\alpha \partial_{t} \mathbf{E}\right), \\
v_{m}=c^{2} / 4 \pi \sigma .
\end{gathered}
$$

Here $\rho$ is the plasma density; $p=p_{i}+p_{e}$ is the plasma pressure (being the sum of the electron and ion pressures); $\mathbf{v}$ is the plasma velocity; $\mathbf{B}$ and $\mathbf{E}$ are the magnetic and electric fields respectively; $\alpha$ is an artificial parameter to control the displacement current; $\mu$ is a gas viscosity; $\varepsilon_{e}$ and $\varepsilon_{i}$ are the internal energies of the electron and ion components of the plasma, respectively, which relate to the unit of the plasma mass; $Q_{e i}$ is the rate of thermal transfer between ions and electrons; $T_{e}$ and $T_{i}$ are the electron and ion temperatures, respectively; $\mathbf{j}$ is the electric current density, $\sigma$ is the electric conductivity; $\kappa_{e}, \kappa_{i}$ are thermal conductivities of electrons and ions, respectively. Analytical matter properties of hydrogen based on Saha's equation as well as dissipation coefficients incorporate the degree of gas ionization [33].

The MHD code MARPLE is the 3D code, used to simulate both stages. The difference scheme approximating the governing MHD system is homogeneous (as to the treatment of shocks and other types of discontinuities) and conservative. The overall time advance procedure is a second order predictor-corrector scheme. The physical model implemented in the code is described in the previous publications [37,38,49] where it was validated with the help of the 1D code NPINCH [33] as well as experimental data [36]. The NPINCH code implements the same model, but in its 1D version, demonstrating a good agreement with the experimental results [50] obtained for the radial electron density distribution in an axially long capillary discharge. Comparison of the simulation results with experimental data in Refs. [27,35,40] gives additional information about applicability of this code to be used to model an additional heating of capillary plasma in order to create proper plasma channel. It is important to stress that the intermediate stages of this additional heating are not onedimensional ones, as it is seen clearly from the simulations [40].

\section{SIMULATION SETUP}

The computational domain of the square cross-section capillary with the side length of $a=500 \mu \mathrm{m}$ is shown in Fig. 2 . The detailed description of the capillary geometry is presented at the end of Sec. II. The electrode is included in the domain together with an additional free space ("outlet") in order to study the plasma outflow from the capillary. The electrode has a circular cross section with the radius of $500 \mu \mathrm{m}$ at the beginning $(0.5<z<0.6) \mathrm{cm}$, widening to $2500 \mu \mathrm{m}$ at its end $(0.6<z<0.7) \mathrm{cm}$.

Hydrogen gas with a pressure of 40 mbar at room temperature is used to fill the capillary (flows throw the inlets marked in Fig. 2). The final steady state is used as the initial state for the discharge simulation. Note that only density distribution from the filling stage is used to set up the initial state of the discharge process. The discharge current is measured experimentally and has the maximum value of $340 \mathrm{~A}$ at around $155 \mathrm{~ns}$ after the beginning of the discharge process (see Fig. 1). The corresponding magnetic field $B(t)=2 I(t) / R$ is defined on the external surface of the insulator as well as on the walls of the supply channels. The boundary conditions at the plasma-electrodes interface as well as the conjunction of the plasma and the insulator subdomains are the same as in [38]. Our previous calculations and comparison with experimental data show that with this approach the time-evolving discharge pattern is reproduced with good accuracy without noticeable losses in the quantitative characteristics of the 


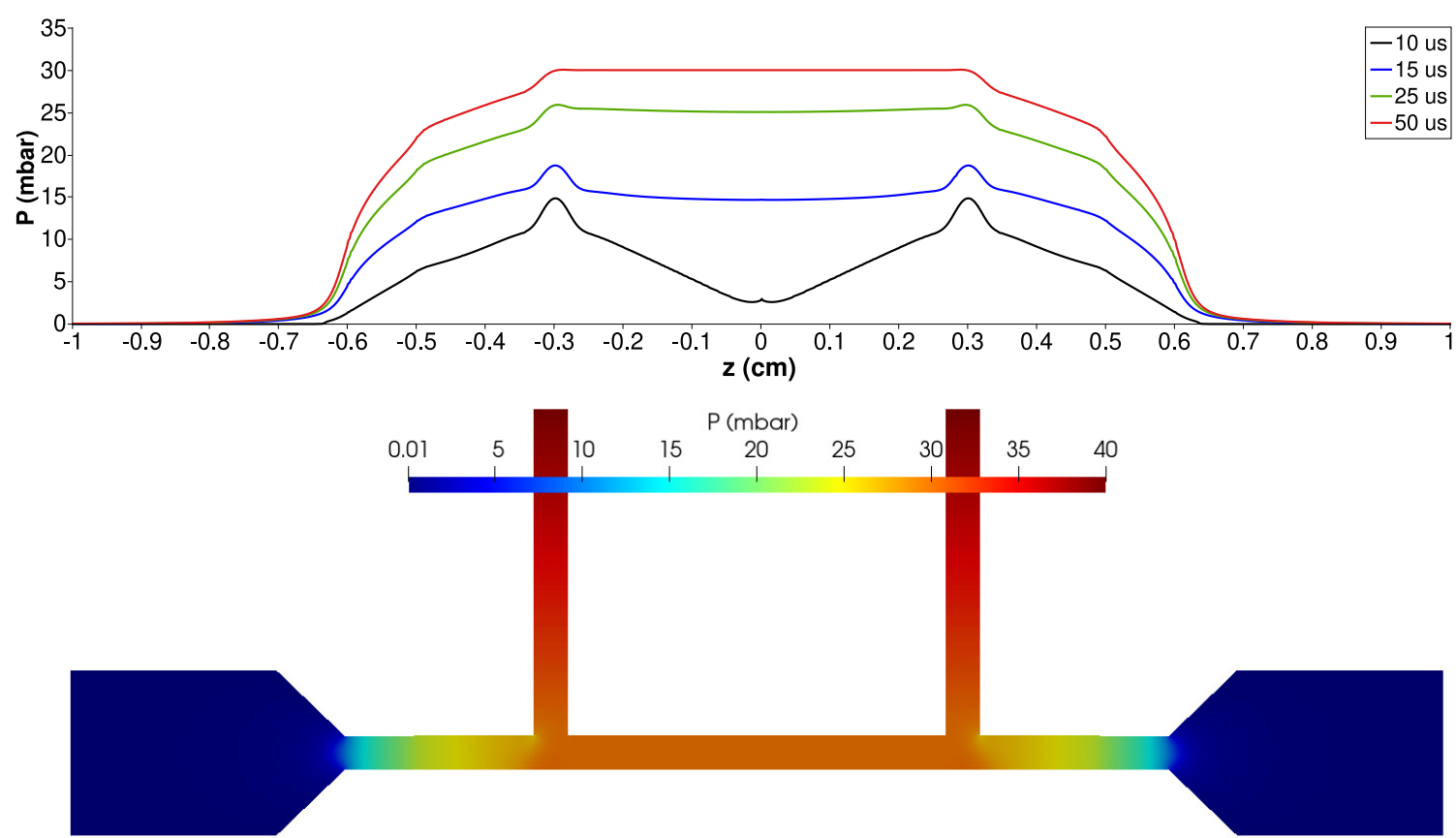

FIG. 3. Axial (grey line at $z=0$ on bottom frame) pressure distributions at several moments of model time (top). Gas pressure distribution at the moment $t=50 \mu$ s (bottom).

discharge plasma. The discharge process is modeled within a $0-300-n s$ time frame.

The additional region around the capillary channel is used for accurate modeling of the magnetic field evolution at the capillary walls as well as in the vicinity of the capillary and supply channel joints. The radius $R$ of this region shall be chosen in such a way that the magnetic field distribution outside of the insulator will be the same as if generated by the current passing through an infinitely thin wire placed instead of the capillary together with supply channels. The value $R=a$, where $a$ is the capillary's transverse size, is suitable for the 2D discharge simulations of square cross-section capillaries [37], however for the 3D simulation we use $R=1.5 \mathrm{a}$ to fulfill the requirement mentioned above in the regions of the capillary and supply channel joint.

For both stages of the simulation (filling and the following discharge) a spatial step $h=10^{-3} \mathrm{~cm}$ (corresponding to 50 cells along the capillary side) is used to discretize the most interesting regions of the domain. For the first stage, the additional region is not needed. Only the quarter of the full domain can be used due to the symmetry which leads to the discretization of $1.9 \times 10^{6}$ cells, while for the second stage half of the full domain is needed as well as the additional insulator region which leads to the discretization of $7.7 \times 10^{6}$ cells.

\section{RESULTS OF THE 3D SIMULATION}

For better understanding the plasma formation mechanism in the case of the electrical discharge in the prefilled capillary channel we perform the 3D simulations of the gas filling and discharge processes in the capillary with predefined parameters.

\section{A. Filling of the capillary with hydrogen}

Gas dynamics of filling the capillary with neutral hydrogen is shown in Fig. 3. One can see that the homogeneous distribution of the gas pressure is formed between supply channels. Outside of them the pressure is smoothly dropped to zero. Note that uniform pressure inside the capillary is less than the inlet pressure (30 mbar instead of 40 mbar) due to the finite gas viscosity which prevents free flow through a narrow channel like supply channels and the capillary itself. Such a drop of the gas density must be taken into account when planning an experiment. The steady state is reached after $50 \mu$ s of filling which is seen in Fig. 4 where evolution

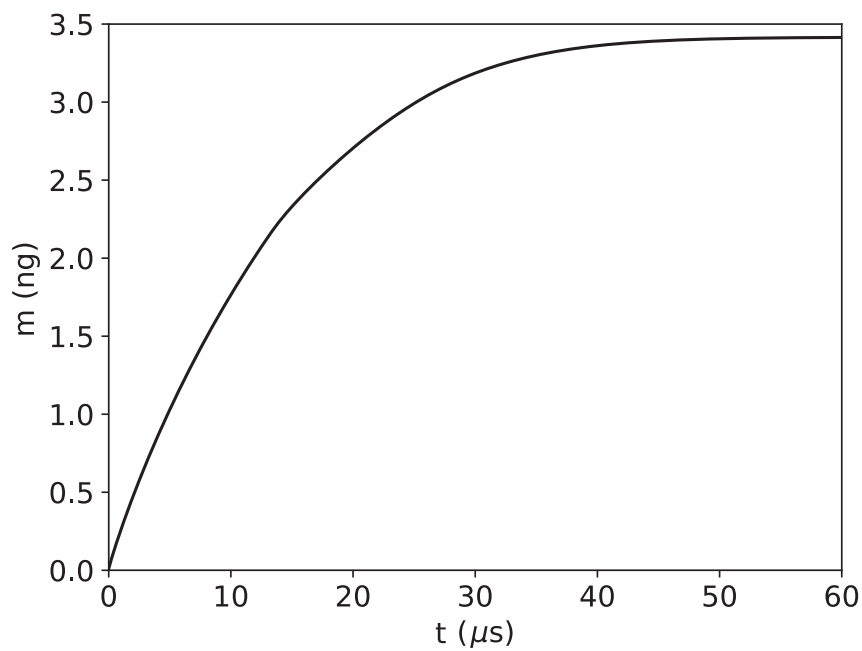

FIG. 4. Total gas mass evolution in the computational domain at filling stage. 


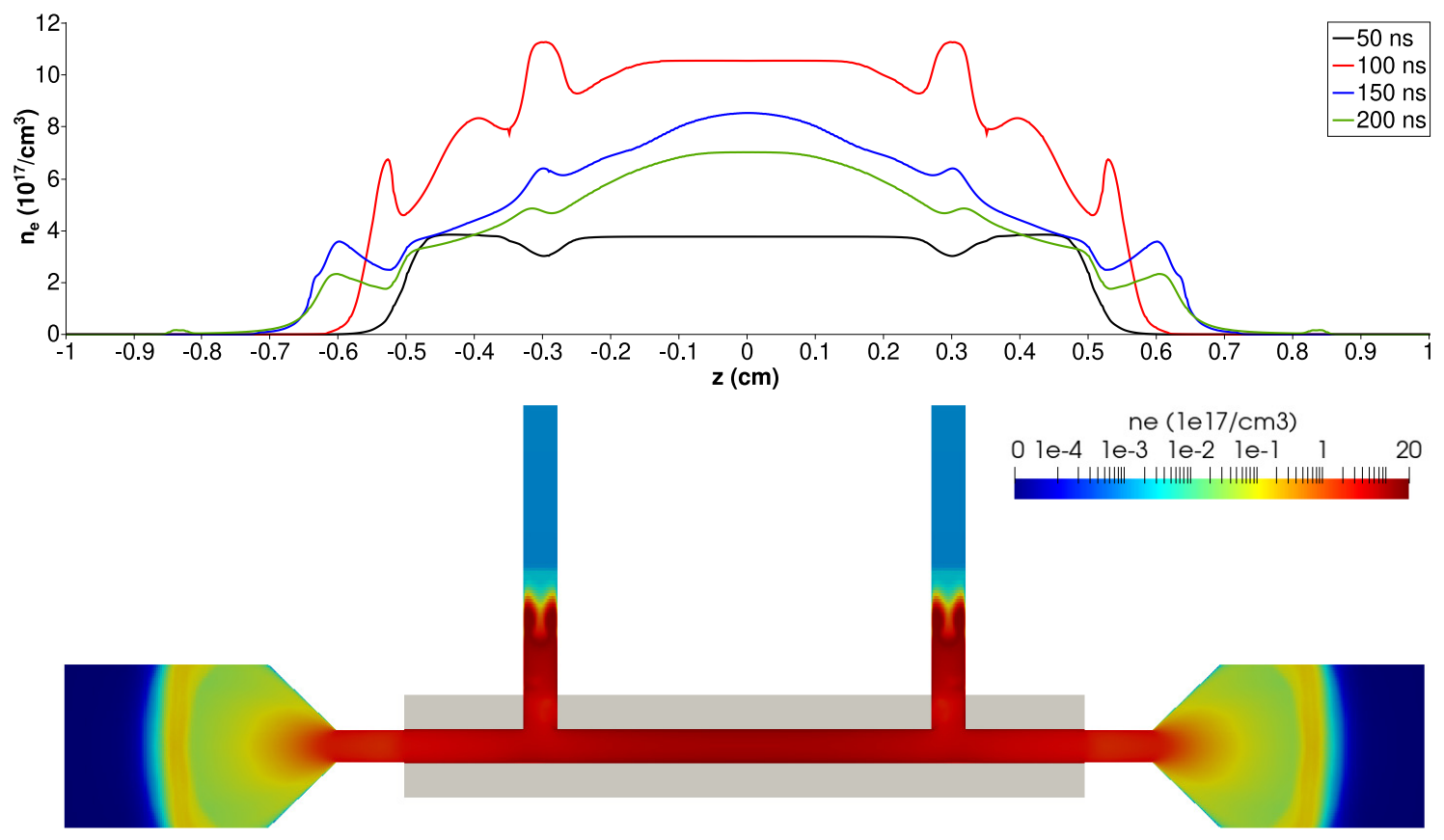

FIG. 5. 2D distribution (bottom) of electron density after $200 \mathrm{~ns}$ of the discharge and its axial (top) profiles at several moments of model time (top).

of total gas mass in the computational domain is shown. By this moment the gas flow rate has reached $0.264 \mathrm{mg} / \mathrm{s}$.

\section{B. Discharge}

Dynamics of the plasma formation (the plasma density distribution) inside the capillary in the case of electrical discharge is shown in Figs. 5 and 6. After approximately $50 \mathrm{~ns}$ from the discharge initiation, the channel is not formed yet and the plasma is still partially ionized-about $50 \%$ in the vicinity of open ends and about $30 \%$ in the middle of the capillary. On the axis, the electron density distribution has small pits in the regions where gas supply channels are placed. In these regions the generated plasma begins to flow back into the channels. At the moment of $100 \mathrm{~ns}$ from the beginning of the electrical discharge the ionization process is almost completed inside the capillary. The ionization level reaches $100 \%$ everywhere except areas in the vicinity of the supply channels where it is around $90 \%$. At this moment, the maximum of the on-axis density is reached, i.e., equilibrium between plasma generation and its outflow is set. The shock waves in both the electrode region and the supply channel region are formed. The stationary shock waves in the joint region of the supply channels and the capillary channel are formed by two opposite plasma flows. One comes from the inner part of the capillary and the other comes from the outer part between the supply channel and the nearest electrode. The longitudinal homogeneity of the plasma channel is reduced to the length of about $0.3 \mathrm{~cm}(z \in[-0.15,+0.15]$ on the top frame in Fig. 7) while its transverse profile still has a noticeable asymmetry, caused by the fact that the plasma from the top part of the capillary, $y>0$, outflows faster to the supply channels than from the bottom part, $y<0$. At the moment when the electric current reaches its maximum
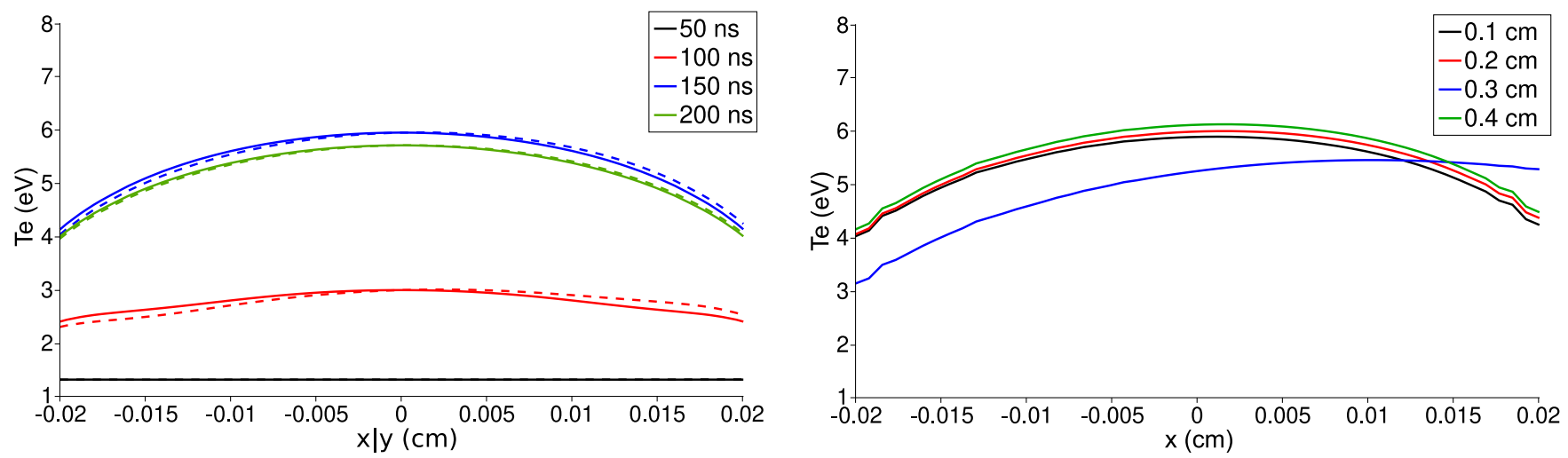

FIG. 6. Transverse electron temperature profiles along (solid) and across (dashed) the capillary axes in the capillary center $(z=0)$ at several moments of modeled time (left) and at several axial positions by $t=150 \mathrm{~ns}$ (right). 

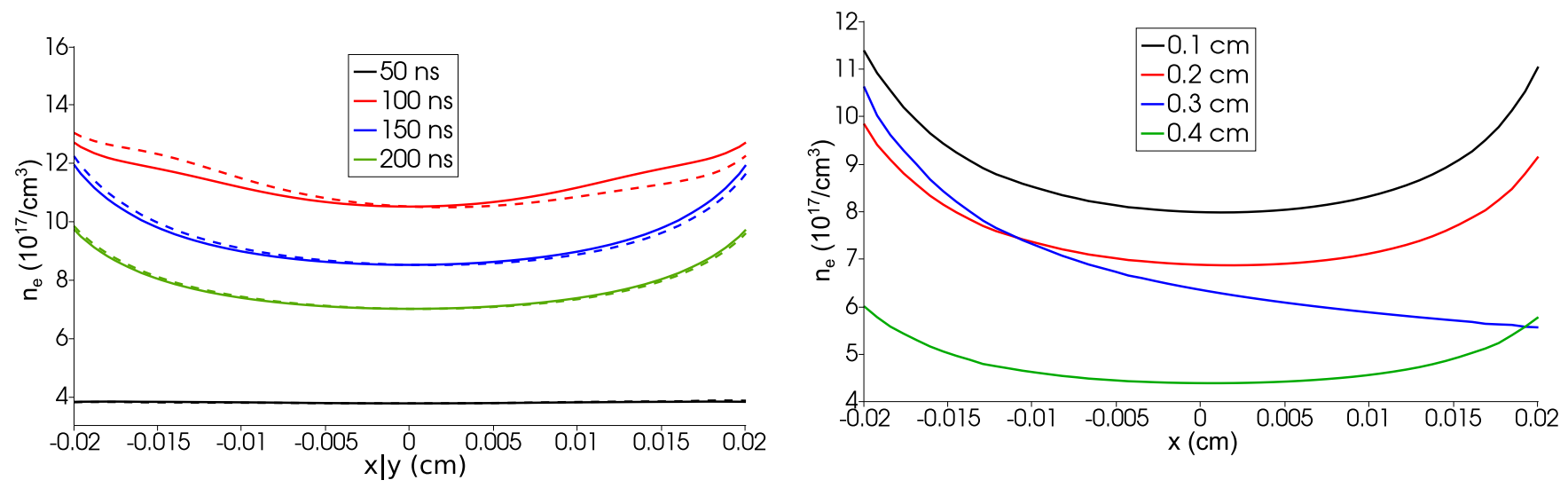

FIG. 7. Transverse electron density profiles along (solid) and across (dashed) the capillary axes in the capillary center $(z=0)$ at several moments of modeled time (left) and at several axial positions by $t=150 \mathrm{~ns}$ (right).

(150 ns) the transverse plasma profile becomes homogeneous in the middle of the capillary (bottom left frame in Fig. 7) however, its longitudinal profile is completely ruined by that moment. The steady shock waves in the vicinity of the supply channels are almost vanished by this time. After $200 \mathrm{~ns}$ of the discharge the plasma starts to outflow from the electrodes; no more shocks in the electron density inside the capillary are observed.

Streamlines of the discharge current in the vicinity of the capillary exits and the supply channel joint points of simulations are presented in Fig. 8. One may notice that the distributions on the bottom left frame are shifted a bit to the left $(y>0)$. It is caused by nonsymmetric geometry of the capillary and hence the plasma outflow to the supply channels. Plasma placed at the top part of the capillary $(y>0)$ outflows faster than plasma in the bottom part; see Fig. 5 where detailed plasma dynamics inside the capillary is presented. Magnetic field distributions at other times are proportional simply to the present electric current shown in Fig. 1. Accuracy of such proportionality is better than $5 \%$ at least at $t \in(100,250) \mathrm{ns}$.

\section{CONCLUSIONS}

We have investigated the capillary discharge plasma channel formation as a part of the laser-driven compact EUV FEL project carried out at ELI-Beamlines. The simulated part of the device includes a square channel inside an insulator, nearest to the channel part of the insulator, gas supplies, electrodes at the ends of the capillary, as well as open volume at the capillary ends, which allows for the plasma to flow out (see Fig. 2).
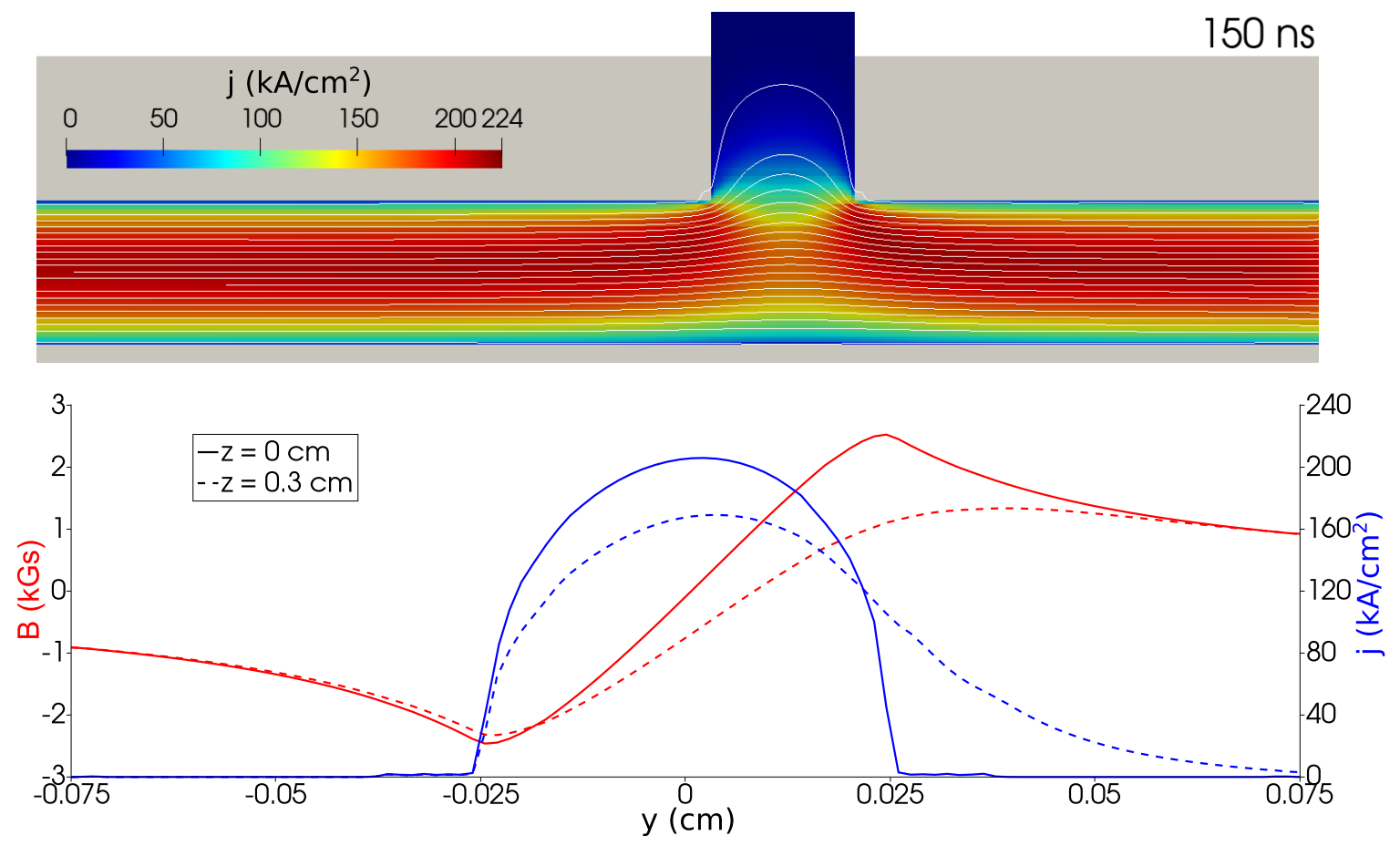

FIG. 8. Discharge current streamlines at the moment of current maximum (top). Magnetic field and discharge current distributions (bottom) in the capillary center (solid) and in the supply channel (dashed). 
The major difference of such a simulation is accounting for the gas supplies which makes electron density and electric current distributions intrinsically three dimensional in the vicinity of the gas inlets.

The distribution of the neutral hydrogen before the onset of the current pulse has been obtained by simulation of the capillary filling process in the same geometry. This simulation also allows us to obtain a relationship between gas consumption and its predischarge pressure. The geometry parameters of the capillary design as well as the initial gas pressure and the parameters of the electric current pulse were chosen to be consistent with the experimental setup.

As the main result, the $3 \mathrm{D}$ distributions of the electron density in the vicinity of the gas suppliers and the electric current density were obtained. This information will allow us to evaluate effects of these disturbances on the processes of the intense laser beam propagation and the electron beam acceleration. This region of the capillary discharge may also affect the quality of the plasma lenses utilizing similar geometry of the capillary. It is worth mentioning that this asymmetry introduced by the analogous gas supplies did not prevent the LWFA acceleration of electron beams in the works by Leemans et al. [26] and Gonsalves et al. [27].

Obtained on-axis electron density distribution at the capillary discharge stage shows formation of the plasma channel suitable for sufficiently distant laser pulse propagation. We conclude that for achieving necessary length of the axially homogeneous plasma channel, in the case of the square shaped capillary with the $500-\mu \mathrm{m}$ size, the capillary has to be lengthened by at least $5 \mathrm{~mm}$, which we will take into consideration to finalize the experimental setup.

The obtained results, discussed above, provide a better understanding of the plasma formation dynamics in the short capillary with the gas channels required to provide a high quality electron beam suitable for the laser-driven compact FEL. The results will be used by the ELI-Beamlines team to analyze the acceleration process, integrating the plasma and particle-in-cell (PIC) simulations. This will demand developing a big data transfer between the codes and multiparametric, joint analysis to find acceptable parameters of the capillary geometry and the plasma and laser beam parameters considering also their time evolution.

\section{ACKNOWLEDGMENTS}

This work was supported by the project High Field Initiative (CZ.02.1.01/0.0/0.0/15_003/0000449) from European Regional Development Fund, by the project "Advanced Research using High Intensity Laser produced Photons and Particles" (ADONIS) (CZ.02.1.01/0.0/0.0/16_019/0000789) from European Regional Development Fund (ERDF) and by the Russian Foundation for Basic Research (Grant No. 20-3170015). Simulations were performed at supercomputer K60 (KIAM RAS).
[1] Y. B. Fainberg, The use of plasma waveguides as accelerating structures in linear accelerators, in CERN Symposium on High Energy Accelerators and Pion Physics (CERN, Geneva, 1956), Vol. 1, pp. 84-90.

[2] T. Tajimaand J. M. Dawson, Laser Electron Accelerator, Phys. Rev. Lett. 43, 267 (1979).

[3] P. Chen, J. M. Dawson, R. W. Huff, and T. Katsouleas, Acceleration of Electrons by the Interaction of a Bunched Electron Beam with a Plasma, Phys. Rev. Lett. 54, 693 (1985).

[4] J. Faure, Y. Glinec, A. Pukhov, S. Kiselev, S. Gordienko, E. Lefebvre, J.-P. Rousseau, F. Burgy, and V. Malka, A laserplasma accelerator producing monoenergetic electron beams, Nature (London) 431, 541 (2004).

[5] S. P. D. Mangles, C. D. Murphy, Z. Najmudin, A. G. R. Thomas, J. L. Collier, A. E. Dangor, E. J. Divall, P. S. Foster, J. G. Gallacher, C. J. Hooker, D. A. Jaroszynski, A. J. Langley, W. B. Mori, P. A. Norreys, F. S. Tsung, R. Viskup, B. R. Walton, and K. Krushelnick, Monoenergetic beams of relativistic electrons from intense laser-plasma interactions, Nature (London) 431 , 535 (2004).

[6] J. Osterhoff, A. Popp, Z. Major, B. Marx, T. P. Rowlands-Rees, M. Fuchs, M. Geissler, R. Hörlein, B. Hidding, S. Becker, E. A. Peralta, U. Schramm, F. Grüner, D. Habs, F. Krausz, S. M. Hooker, and S. Karsch, Generation of Stable, LowDivergence Electron Beams by Laser-Wakefield Acceleration in a Steady-State-Flow Gas Cell, Phys. Rev. Lett. 101, 085002 (2008).

[7] J. S. Liu, C. Q. Xia, W. T. Wang, H. Y. Lu, C. Wang, A. H. Deng, W. T. Li, H. Zhang, X. Y. Liang, Y. X. Leng, X. M.
Lu, C. Wang, J. Z. Wang, K. Nakajima, R. X. Li, and Z. Z. $\mathrm{Xu}$, All-Optical Cascaded Laser Wakefield Accelerator Using Ionization-Induced Injection, Phys. Rev. Lett. 107, 035001 (2011).

[8] F. Grüner, S. Becker, U. Schramm, T. Eichner, M. Fuchs, R. Weingartner, D. Habs, J. Meyer-ter Vehn, M. Geissler, M. Ferrario, L. Serafini, B. van der Geer, H. Backe, W. Lauth, and S. Reiche, Design considerations for table-top, laser-based VUV and X-ray free electron lasers, Appl. Phys. B 86, 431 (2007).

[9] M. Fuchs, R. Weingartner, A. Popp, Z. Major, S. Becker, J. Osterhoff, I. Cortrie, B. Zeitler, R. Hörlein, G. D. Tsakiris, U. Schramm, T. P. Rowlands-Rees, S. M. Hooker, D. Habs, F. Krausz, S. Karsch, and F. Grüner, Laser-driven soft-x-ray undulator source, Nat. Phys. 5, 826 (2009).

[10] Z. Huang, Y. Ding, and C. B. Schroeder, Compact X-Ray Free-Electron Laser from a Laser-Plasma Accelerator Using a Transverse-Gradient Undulator, Phys. Rev. Lett. 109, 204801 (2012).

[11] M. E. Couprie, A. Loulergue, M. Labat, R. Lehe, and V. Malka, Towards a free electron laser based on laser plasma accelerators, J. Phys. B 47, 234001 (2014).

[12] A. R. Maier, A. Meseck, S. Reiche, C. B. Schroeder, T. Seggebrock, and F. Grüner, Demonstration Scheme for a LaserPlasma-Driven Free-Electron Laser, Phys. Rev. X 2, 031019 (2012).

[13] A. Molodozhentsev, G. Korn, A. Maier, and L. Pribyl, LWFA-driven Free Electron Laser for ELI-Beamlines, in Proceedings of the 60th ICFA Advanced Beam Dynamics Workshop 
(FLS'18), Shanghai, China, 5-9 March 2018, ICFA Advanced Beam Dynamics Workshop No. 60 (JACoW, Geneva, 2018), pp. 62-67.

[14] R. W. Assmann, M. K. Weikum, T. Akhter, D. Alesini, A. S. Alexandrova, M. P. Anania, N. E. Andreev, I. Andriyash, M. Artioli, A. Aschikhin et al., Eupraxia conceptual design report, Eur. Phys. J.: Spec. Top. 229, 3675 (2020).

[15] K. Ta Phuoc, S. Corde, C. Thaury, V. Malka, A. Tafzi, J. P. Goddet, R. C. Shah, S. Sebban, and A. Rousse, All-optical compton gamma-ray source, Nat. Photonics 6, 308 (2012).

[16] C. G. R. Geddes, S. Rykovanov, N. H. Matlis, S. Steinke, J.-L. Vay, E. H. Esarey, B. Ludewigt, K. Nakamura, B. J. Quiter, C. B. Schroeder, C. Toth, and W. P. Leemans, Compact quasi-monoenergetic photon sources from laser-plasma accelerators for nuclear detection and characterization, Nucl. Instrum. Methods. Phys. Res. B 350, 116 (2015).

[17] K. Khrennikov, J. Wenz, A. Buck, J. Xu, M. Heigoldt, L. Veisz, and S. Karsch, Tunable All-Optical Quasimonochromatic Thomson X-Ray Source in the Nonlinear Regime, Phys. Rev. Lett. 114, 195003 (2015).

[18] W. Leemans and E. Esarey, Laser-driven plasma-wave electron accelerators, Phys. Today 62(3), 44 (2009).

[19] C. B. Schroeder, E. Esarey, C. G. R. Geddes, C. Benedetti, and W. P. Leemans, Physics considerations for laser-plasma linear colliders, Phys. Rev. ST: Accel. Beams 13, 101301 (2010).

[20] T. Hosokai, M. Kando, H. Dewa, H. Kotaki, S. Kondo, N. Hasegawa, K. Nakajima, and K. Horioka, Optical guidance of terrawatt laser pulses by the implosion phase of a fast $\mathrm{z}$-pinch discharge in a gas-filled capillary, Opt. Lett. 25, 10 (2000).

[21] A. Butler, D. J. Spence, and S. M. Hooker, Guiding of High-Intensity Laser Pulses with a Hydrogen-Filled Capillary Discharge Waveguide, Phys. Rev. Lett. 89, 185003 (2002).

[22] D. J. Spence, A. Butler, and S. M. Hooker, Gas-filled capillary discharge waveguides, J. Opt. Soc. Am. B 20, 138 (2003).

[23] S. Karsch, J. Osterhoff, A. Popp, T. P. Rowlands-Rees, Z. Major, M. Fuchs, B. Marx, R. Hörlein, K. Schmid, L. Veisz, S. Becker, U. Schramm, B. Hidding, G. Pretzler, D. Habs, F. Grüner, F. Krausz, and S. M. Hooker, GeV-scale electron acceleration in a gas-filled capillary discharge waveguide, New J. Phys. 9, 415 (2007).

[24] T. Kameshima, H. Kotaki, M. Kando, I. Daito, K. Kawase, Y. Fukuda, L. M. Chen, T. Homma, S. Kondo, T. Z. Esirkepov, N. A. Bobrova, P. V. Sasorov, and S. V. Bulanov, Laser pulse guiding and electron acceleration in the ablative capillary discharge plasma, Phys. Plasmas 16, 093101 (2009).

[25] A. J. Gonsalves, K. Nakamura, C. Lin, D. Panasenko, S. Shiraishi, T. Sokollik, C. Benedetti, C. B. Schroeder, C. G. R. Geddes, J. van Tilborg, J. Osterhoff, E. Esarey, C. Toth, and W. P. Leemans, Tunable laser plasma accelerator based on longitudinal density tailoring, Nat. Phys. 7, 862 (2011).

[26] W. P. Leemans, A. J. Gonsalves, H.-S. Mao, K. Nakamura, C. Benedetti, C. B. Schroeder, C. Tóth, J. Daniels, D. E. Mittelberger, S. S. Bulanov, J.-L. Vay, C. G. R. Geddes, and E. Esarey, Multi-Gev Electron Beams from Capillary-DischargeGuided Subpetawatt Laser Pulses in the Self-Trapping Regime, Phys. Rev. Lett. 113, 245002 (2014).

[27] A. J. Gonsalves, K. Nakamura, J. Daniels, C. Benedetti, C. Pieronek, T. C. H. de Raadt, S. Steinke, J. H. Bin, S. S. Bulanov, J. van Tilborg, C. G. R. Geddes, C. B. Schroeder, C. Tóth, E. Esarey, K. Swanson, L. Fan-Chiang, G. Bagdasarov, N.
Bobrova, V. Gasilov, G. Korn et al., Petawatt Laser Guiding and Electron Beam Acceleration to $8 \mathrm{GeV}$ in a Laser-Heated Capillary Discharge Waveguide, Phys. Rev. Lett. 122, 084801 (2019).

[28] W. K. H. Panofsky and W. R. Baker, A focusing device for the external $350-\mathrm{MeV}$ proton beam of the 184-inch cyclotron at berkeley, Rev. Sci. Instrum. 21, 445 (1950).

[29] J. van Tilborg, S. Steinke, C. G. R. Geddes, N. H. Matlis, B. H. Shaw, A. J. Gonsalves, J. V. Huijts, K. Nakamura, J. Daniels, C. B. Schroeder, C. Benedetti, E. Esarey, S. S. Bulanov, N. A. Bobrova, P. V. Sasorov, and W. P. Leemans, Active Plasma Lensing for Relativistic Laser-Plasma-Accelerated Electron Beams, Phys. Rev. Lett. 115, 184802 (2015).

[30] A. Ferran Pousa, A. Martinez de la Ossa, R. Brinkmann, and R. W. Assmann, Compact Multistage Plasma-Based Accelerator Design for Correlated Energy Spread Compensation, Phys. Rev. Lett. 123, 054801 (2019).

[31] Y. Ehrlich, C. Cohen, A. Zigler, J. Krall, P. Sprangle, and E. Esarey, Guiding of High Intensity Laser Pulses in Straight and Curved Plasma Channel Experiments, Phys. Rev. Lett. 77, 4186 (1996).

[32] D. J. Spence and S. M. Hooker, Investigation of a hydrogen plasma waveguide, Phys. Rev. E 63, 015401(R) (2000).

[33] N. A. Bobrova, A. A. Esaulov, J.-I. Sakai, P. V. Sasorov, D. J. Spence, A. Butler, S. M. Hooker, and S. V. Bulanov, Simulations of a hydrogen-filled capillary discharge waveguide, Phys. Rev. E 65, 016407 (2001).

[34] N. A. Bobrova, P. V. Sasorov, C. Benedetti, S. S. Bulanov, C. G. R. Geddes, C. B. Schroeder, E. Esarey, and W. P. Leemans, Laser-heater assisted plasma channel formation in capillary discharge waveguides, Phys. Plasmas 20, 020703 (2013).

[35] A. J. Gonsalves, K. Nakamura, C. Benedetti, C. V. Pieronek, S. Steinke, J. H. Bin, S. S. Bulanov, J. van Tilborg, C. G. R. Geddes, C. B. Schroeder, J. Daniels, C. Tóth, L. Obst-Huebl, R. G. W. van den Berg, G. Bagdasarov, N. Bobrova, V. Gasilov, G. Korn, P. Sasorov, W. P. Leemans et al., Laser-heated capillary discharge plasma waveguides for electron acceleration to 8 gev, Phys. Plasmas 27, 053102 (2020).

[36] C. V. Pieronek, A. J. Gonsalves, C. Benedetti, S. S. Bulanov, J. van Tilborg, J. H. Bin, K. K. Swanson, J. Daniels, G. A. Bagdasarov, N. A. Bobrova, V. A. Gasilov, G. Korn, P. V. Sasorov, C. G. R. Geddes, C. B. Schroeder, W. P. Leemans, and E. Esarey, Laser-heated capillary discharge waveguides as tunable structures for laser-plasma acceleration, Phys. Plasmas 27, 093101 (2020).

[37] G. Bagdasarov, P. Sasorov, A. Boldarev, O. Olkhovskaya, V. Gasilov, A. J. Gonsalves, S. Barber, S. S. Bulanov, C. B. Schroeder, J. van Tilborg, E. Esarey, W. P. Leemans, T. Levato, D. Margarone, G. Korn, and S. V. Bulanov, Plasma equilibrium inside various cross-section capillary discharges, Phys. Plasmas 24, 053111 (2017).

[38] G. A. Bagdasarov, P. V. Sasorov, V. A. Gasilov, A. S. Boldarev, O. G. Olkhovskaya, C. Benedetti, S. S. Bulanov, A. Gonsalves, H.-S. Mao, C. B. Schroeder, J. van Tilborg, E. Esarey, W. P. Leemans, T. Levato, D. Margarone, and G. Korn, Laser beam coupling with capillary discharge plasma for laser wakefield acceleration applications, Phys. Plasmas 24, 083109 (2017).

[39] E. Brentegani, M. Anania, S. Atzeni, A. Biagioni, E. Chiadroni, M. Croia, M. Ferrario, F. Filippi, A. Marocchino, A. Mostacci, 
R. Pompili, S. Romeo, A. Schiavi, and A. Zigler, Numerical studies on capillary discharges as focusing elements for electron beams, Nucl. Instrum. Methods Phys. Res. A 909, 404 (2018), 3rd European Advanced Accelerator Concepts workshop (EAAC2017).

[40] G. A. Bagdasarov, N. A. Bobrova, O. G. Olkhovskaya, V. A. Gasilov, C. Benedetti, S. S. Bulanov, A. J. Gonsalves, C. V. Pieronek, J. van Tilborg, C. G. R. Geddes, C. B. Schroeder, P. V. Sasorov, S. V. Bulanov, G. Korn, and E. Esarey, Creation of an axially uniform plasma channel in a laser-assisted capillary discharge, Phys. Plasmas 28, 053104 (2021).

[41] V. Gasilov, A. Boldarev, S. Dyachenko, O. Olkhovskaya, E. Kartasheva, G. Bagdasarov, S. Boldyrev, I. Gasilova, V. Shmyrov, S. Tkachenko, J. Grunenwald, and T. Maillard, Towards an application of high-performance computer systems to 3D simulations of high energy density plasmas in Z-Pinches, in Applications, Tools and Techniques on the Road to Exascale Computing, Advances in Parallel Computing Vol. 22 (IOS Press BV, Amsterdam, 2012), pp. 235-242.

[42] A. Molodozhentsev, J. Green, J. Hawke, M. Kaur, D. Kocon, G. Korn, K. Kruchinin, and A. Maier, Laser-Driven Compact Free Electron Laser Development at ELI-Beamlines, in Proceedings of FEL'19, Free Electron Laser Conference No. 39 (JACoW, Geneva, 2019), pp. 680-683.

[43] T. P. Rowlands-Rees, C. Kamperidis, S. Kneip, A. J. Gonsalves, S. P. D. Mangles, J. G. Gallacher, E. Brunetti, T. Ibbotson, C. D. Murphy, P. S. Foster, M. J. V. Streeter, F. Budde, P. A. Norreys, D. A. Jaroszynski, K. Krushelnick, Z. Najmudin, and S. M. Hooker, Laser-Driven Acceleration of Electrons in a Partially Ionized Plasma Channel, Phys. Rev. Lett. 100, 105005 (2008).
[44] T. Schmidt, P. Böhler, M. Brügger, M. Calvi, S. Danner, P. Huber, A. Imhof, H. Jöhri, A. Keller, M. Locher, T. Stapf, and J. Wickstroem, in Proceedings of FEL2012 (JACoW, Geneva, 2012), pp. 666-669.

[45] A. Molodozhentsev and K. O. Kruchinin, Compact LWFABased Extreme Ultraviolet Free Electron Laser: design constraints, Instruments 6, 4 (2022).

[46] W. Wang, K. Feng, L. Ke, C. Yu, Y. Xu, R. Qi, Y. Chen, Z. Qin, Z. Zhang, M. Fang, J. Liu, K. Jiang, H. Wang, C. Wang, X. Yang, F. Wu, Y. Leng, J. Liu, R. Li, and Z. Xu, Free-electron lasing at 27 nanometres based on a laser wakefield accelerator, Nature (London) 595, 516 (2021).

[47] W. Lu, M. Tzoufras, C. Joshi, F. S. Tsung, W. B. Mori, J. Vieira, R. A. Fonseca, and L. O. Silva, Generating multi-gev electron bunches using single stage laser wakefield acceleration in a $3 \mathrm{~d}$ nonlinear regime, Phys. Rev. ST: Accel. Beams 10, 061301 (2007).

[48] S. I. Braginskii, Review of Plasma Physics (Consultant Bureau, New York, 1965), Vol. 1.

[49] O. G. Olkhovskaya, G. A. Bagdasarov, N. A. Bobrova, V. A. Gasilov, L. V. N. Goncalves, C. M. Lazzarini, M. Nevrkla, G. Grittani, S. S. Bulanov, A. J. Gonsalves, C. B. Schroeder, E. Esarey, W. P. Leemans, P. V. Sasorov, S. V. Bulanov, and G. Korn, Plasma channel formation in the knife-like focus of laser beam, J. Plasma Phys. 86, 905860307 (2020).

[50] M. Turner, A. J. Gonsalves, S. S. Bulanov, C. Benedetti, N. A. Bobrova, V. A. Gasilov, P. V. Sasorov, G. Korn, K. Nakamura, J. van Tilborg, C. G. Geddes, C. B. Schroeder, and E. Esarey, Radial density profile and stability of capillary discharge plasma waveguides of lengths up to $40 \mathrm{~cm}$, High Power Laser Sci. Eng. 9, e17 (2021) 